\title{
Stock Price Trend Prediction Model Based on WNN with Redundant Structure Reduced by RS
}

\author{
Ren Shuili \\ XiJing College \\ Xi'an, Shaanxi, China 710123 \\ ganxusheng123@163.com
}

\author{
Lou Yuanwei \\ Air Traffic Control and Navigation College \\ Air Force Engineering University \\ Xi'an, Shaanxi, 710051, China \\ 911yhk@163.com
}

\author{
Lei Lei \\ School of Business Administration \\ Henan University of Economics and Law \\ Zhengzhou, Henan, 050046, China \\ lyx521@yahoo.com.cn
}

\begin{abstract}
Due to lots of factors affecting the fluctuation of stock price, it is very difficult to the accurately predict the stock price. For this problem, a Wavelet Neural Network (WNN) stock price prediction method is proposed, and Rough Set (RS) method is introduced to reduce the Input dimensions of $\mathrm{WNN}$ and optimize the hidden layer nodes of WNN for optimization structure reduction. The experiment results show that, the introduction of RS attributes reduction can simplify the structure of WNN model can be to a great extent for stock price trend with improvement of the performance. The direction symmetry of prediction corresponding to SSE Composite Index, CSI 300 Index and All Ordinaries Index is $65.75 \%, 66.37 \%$ and $65.93 \%$ with $1.7 \mathrm{~s}, 1.8 \mathrm{~s}$ and $2.1 \mathrm{~s}$ training time, respectively. The prediction result is better than that of other neural network and WNN models.
\end{abstract}

Keywords-Wavelet Neural Network; Rough Set; Stock Price Prediction; Structure Optimization

\section{INTRODUCTION}

Using Neural Network (NN) to predict the stock price has many difficulties because of the uncertain interaction between these influence factors [1][2]. Compared with the traditional NN, Wavelet Neural Network (WNN) has a great improvement in strict theory and good mapping capability, suitable for all areas of neural network application [3][4]. However, to accurately predict the stock price use using WNN, some problems such as input dimension reduction and structure optimization in WNN application need to be solved. Based on it, a WNN prediction method that use Rough Set (RS) attribute reduction [5][6] to conduct the input dimension reduction and structure optimization is proposed for stock price. The feasibility of this method is proved by the experiences.

\section{WAVELET NeUral Network}

WNN is a novel NN developed on the basis of wavelet transform. Because the wavelet basis is used as the activation function to replace the usual neuron, and the excellent time-frequency localization properties of wavelet transform is integrated with the self learning function of traditional NN, WNN shows the strong approximation and tolerance. The structure of WNN with a typical three-layer structure is shown in Fig. 1.

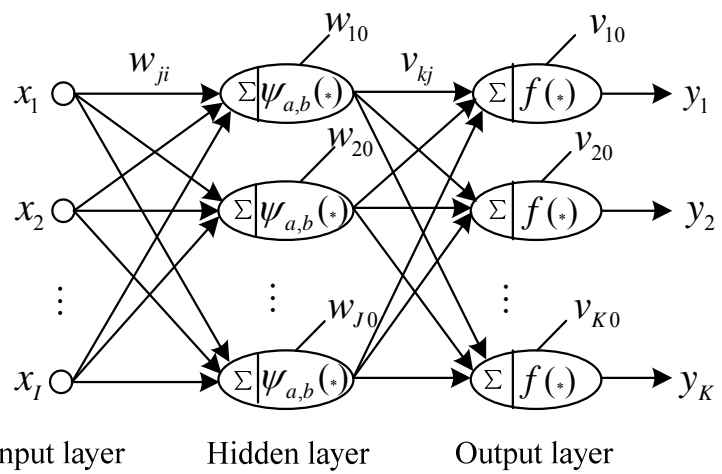

Fig. 1. Structure of WNN

In Fig. $1, \quad x_{i}(i=1, \cdots, I)$ is the input; $y_{k}(k=1$, $\cdots, K)$ is the output; $w_{j i}(i=1, \cdots, I ; j=1, \cdots, J)$ is the connection weight between input and hidden layer; $w_{j 0}(j=1, \cdots, J)$ is the threshold of hidden layer node; $v_{k j}(j=1, \cdots ; J ; k=1, \cdots ; K)$ is the connection weight between hidden and output layer; $v_{k 0}(k=1, \cdots, K)$ is the threshold of output layer node. $I$ is the number of input nodes; $J$ is the number of hidden nodes; $K$ is the number of output nodes. $\psi_{a, b}(\cdot)$ is wavelet basis function of hidden layer. In this paper, Morlet wavelet function $\psi_{a, b}(t)=\cos (1.75 t) e^{-t^{2} / 2}$ is adopted, where $a, b$ is the dilation and translation factor 
respectively. $f(\cdot)$ is the activation function of output layer which can be taken as Sigmoid function: $f(t)=1 /\left(1+e^{-t}\right)$.

\section{WNN MODELING REALIZATION OF DIMENSION REDUCTION AND NETWORK OPTIMIZATION CONDUCTED BY RS}

For the unsatisfactory WNN modeling result caused by the large input dimension and complex network structure, RS is introduced to reduce the complexity of WNN modeling and structure. RS can reduce the overall attribute number under the premise of keeping the same classification ability, and has become the core research contents of RS theory, which can remove the redundant structure of $\mathrm{WNN}$ for reasonable network [7][8]. The modeling process is shown in Fig. 2. In the flow, first, RS is used to reduce the input dimension of WNN, and then RS is applied to optimize the network structure of WNN [9].

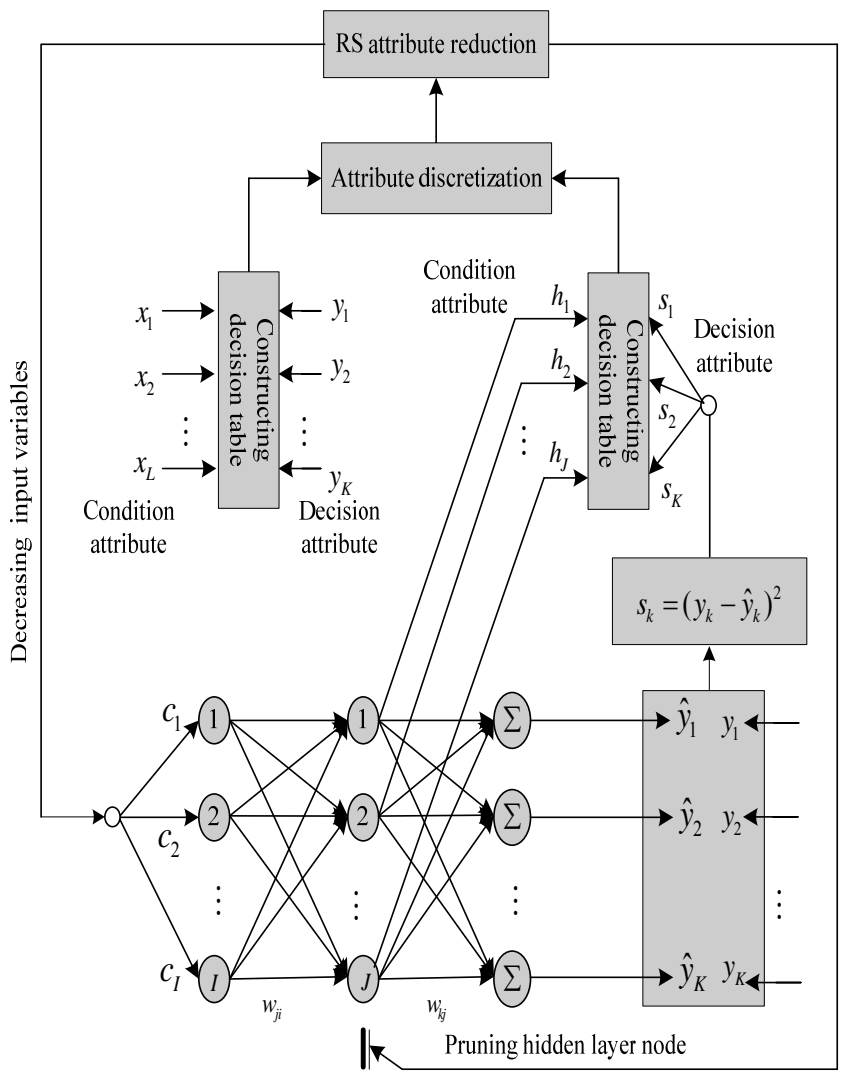

Fig. 2. Integration principle of RS-WNN

\section{SELECTION OF EXPERIMENT DATA}

To validate the performance of proposed model, all experiment data used is from 3 famous stock indexes: SSE Composite Index (China), CSI 300 Index (China) and All Ordinaries Index (Australian). The original data is respectively in the range of 4/10/2009 6/24/2014 from SSE Composite Index, 2/9/2009 4/2/2014 from CSI 300 Index, 4/21/2009 3/26/2014 from All Ordinaries Index (www.resset.cn). The original data include: opening price, top price, bottom price, closing price and trading volume. To facilitate the modeling and testing, the original data need to be transformed into 15 index feature quantities as shown in Table 1 in advance by calculation formula, and these index feature quantities are used as the inputs of the prediction model to be established. In addition, the prediction output of the model is the change trend of stock price. According to the percent yield, its change trends can be defined as: Falling: $R \in(-\infty,-0.5 \%$ ], Invariability: $R \in(-0.5 \%, 0.5 \%)$ and Climbing: $R \in[0.5 \%, \infty), R$ denotes the percent yield that can be calculated by

$$
R_{t}=\left(P_{t}-P_{t-1}\right) / P_{t-1} \times 100 \%
$$

where $P_{t}$ is the series of stock price.

Index feature illustration:

1. Random \%K: RSVt $=100 \times(\mathrm{Ct}-\mathrm{Ln}) /(\mathrm{Hn}-\mathrm{Ln}), \mathrm{Ct}$ is the day closing price, $\mathrm{Ln}$ and $\mathrm{Hn}$ is the lowest price and highest price within the cycle respectively.

2. Random $\% \mathrm{D}$ : It is the smoothed moving average line of $\% \mathrm{~K}, \% \mathrm{~K}$ and $\% \mathrm{D}$ cycle can be taken as 5 days.

3. W \& R: Ln and Hn is the lowest price and highest price within the cycle respectively, $\mathrm{Cn}$ is the day closing price, the cycle $\mathrm{n}$ can be taken as 5 days.

4. MTM: Ct, Ct-n is the day closing price and first $\mathrm{n}$ day closing price respectively, the cycle $\mathrm{n}$ can be taken as 5 days.

TABLE I. INDEX FEATURE QUANTITY AND ITS CALCULATION FORMULA

\begin{tabular}{|c|c|c|}
\hline ID & $\begin{array}{c}\text { Index feature } \\
\text { quantity }\end{array}$ & Calculation formula \\
\hline 1 & Random \%K & $1 / 3 \times \mathrm{RSV}_{\mathrm{t}}+2 / 3 \times \% \mathrm{tK}_{\mathrm{t}-1}$ \\
\hline 2 & Random \%D & $1 / 3 \times \% \mathrm{~K}_{\mathrm{t}}+2 / 3 \times \% \mathrm{D}_{\mathrm{t}-1}$ \\
\hline 3 & $\mathrm{~W} \& \mathrm{R}$ & $100 \times\left(H_{n}-C_{n}\right) /\left(H_{n}-L_{n}\right)$ \\
\hline 4 & МТМ & $C_{t}-C_{t-n}$ \\
\hline 5 & RSI & $100 \times \Sigma U_{n} /\left(\Sigma U_{n}+\Sigma D P_{n}\right)$ \\
\hline 6 & BBI & $\left(\mathrm{MA}_{3}+\mathrm{MA}_{6}+\mathrm{MA}_{12}+\mathrm{MA}_{24}\right) / 4$ \\
\hline 7 & 5BIAS & $100 \times\left(\mathrm{C}_{\mathrm{t}}-\mathrm{MA}_{5}\right) / \mathrm{MA}_{5}$ \\
\hline 8 & 10BIAS & $100 \times\left(\mathrm{C}_{\mathrm{t}}-\mathrm{MA}_{10}\right) / \mathrm{MA}_{10}$ \\
\hline 9 & MB & $\mathrm{MA}_{3}-\mathrm{MA}_{6}$ \\
\hline 10 & PSY & $100 \times \mathrm{DUP}_{\mathrm{n}} / \mathrm{n}$ \\
\hline 11 & AR & $100 \times \mathrm{HO}_{\mathrm{n}} / \mathrm{OL}_{\mathrm{n}}$ \\
\hline 12 & $\mathrm{BR}$ & $100 \times \mathrm{H} \mathrm{C}_{\mathrm{n}} / \mathrm{C} \mathrm{L}_{\mathrm{n}}$ \\
\hline 13 & MV & $\left(V_{1}+V_{2}+\ldots+V_{n}\right) / n$ \\
\hline 14 & TAPI & $\mathrm{V}_{\mathrm{n}} / \mathrm{C}_{\mathrm{n}}$ \\
\hline 15 & VR & $100 \times \Sigma V$ Upn $/ \Sigma V_{n}$ \\
\hline
\end{tabular}


5. RSI: $\Sigma U P_{n}$ is the sum of the rising of daily closing price within the cycle, $\Sigma \mathrm{DW}_{\mathrm{n}}$ is the absolute value of the sum of the falling of daily closing price within the cycle, the cycle $\mathrm{n}$ can be taken as 5 days.

6. BBI: It is the mixed moving average, MA3, MA6, MA12, MA24 denotes the moving average within $3,6,12,24$ days respectively.

7. 5BIAS: $\mathrm{Ct}$ is the day closing price, MA5 denotes the moving average within 5 days.

8. 10BIAS: $\mathrm{Ct}$ is the day closing price, MA10 denotes the moving average within 10 days.

9. MB: MA3, MA6 denotes the moving average within 3 and 6 days respectively.

10. PSY: DUPn denotes the number of rising days within the selected cycle; the cycle $\mathrm{n}$ can be taken as 10 days.

11. AR: HOn is the sum of distance from highest price to opening price within $\mathrm{n}$ days, OLn is the sum of distance from opening price to lowest price within $\mathrm{n}$ days.

12. BR: $\mathrm{HCn}$ is the sum of distance from highest price to closing price within $\mathrm{n}$ days, CLn is the sum of distance from closing price to lowest price within $\mathrm{n}$ days.

13. MV: V1, V2,.., Vn denotes the daily trading volume within a given cycle, the cycle $\mathrm{n}$ can be taken as 5 days.

14. TAPI: $\mathrm{Vn}$ is the day trading volume, $\mathrm{Cn}$ is the day closing price.

15. $\Sigma$ VUPn is the sum of rising daily trading volume within the cycle, $\Sigma \mathrm{V}_{\mathrm{n}}$ is the total volume within the cycle, the cycle $\mathrm{n}$ can be taken as 5 days.

\section{EXPERIMENT RESULT ANALYSIS}

Experimental scheme: Compare with other models to validate the effectiveness of $2 \mathrm{RS}-\mathrm{WNN}$ prediction model for stock price trend. First, 600 samples are randomly selected from the data of SSE Composite Index (China), CSI 300 Index (China) and All Ordinaries Index (Australian) respectively to construct the training set with 500 samples and test set with 100 samples. Then the stock price trend prediction model based on BP-NN, RBF-NN, ANFIS-NN, WNN, RS-WNN, $2 \mathrm{RS}-\mathrm{WNN}$ is established and is tested, respectively. Finally the performances of built models are compared to validate the superiority of $2 \mathrm{RS}-\mathrm{WNN}$ in stock price trend prediction. $w_{j i}, v_{k j}, w_{j 0}, v_{k 0}, a_{j}, b_{j}$ can be initialized as the random number in $[0,1]$, the maximum training number can be taken as 200. In the experiment, except for 2RS-WNN, the number of hidden nodes of BP-NN, RBF-NN, ANFIS-NN, WNN and RS-WNN all use Exhaustive Attack Method (EAM) to determine.

Through the analysis and study on relevant literature, seven evaluation indices are determined to evaluate the performance of built models including: Root Mean Square Error (RMSE), Mean Absolute Difference (MAD), Mean Absolute Percentage Error (MAPE); Directional Symmetry (DS),Correct Uptrend (CP) and Correct Downtrend (CD). RMSE, MAD and MAPE are used to measure the deviation between actual value and predicted value. DS, CP and CD is applied to measure the accuracy of the prediction. DS is used to describe the accuracy of stock index direction prediction in percentage form. $\mathrm{CP}$ is used to describe the accuracy of stock index direction prediction for the upward trend in percentage form. CD is used to describe the accuracy of stock index direction prediction for the downward trend in percentage form. The formula of DS, $\mathrm{CP}$ and $\mathrm{CD}$ are as follows:

$$
\begin{gathered}
D S=\frac{100}{n} \sum_{i=1}^{n} d_{i}, \\
d_{i}= \begin{cases}1 & \left(P_{i}-P_{i-1}\right)\left(T_{i}-T_{i-1}\right) \geq 0 \\
0 & \text { Other }\end{cases} \\
C P=\frac{100}{n_{1}} \sum_{i=1}^{n} d_{i}, \\
d_{i}= \begin{cases}1 & \left(T_{i}-T_{i-1}\right)>0 \text { and }\left(P_{i}-P_{i-1}\right)\left(T_{i}-T_{i-1}\right) \geq 0 \\
0 & \text { Other }\end{cases} \\
d_{i}=\left\{\begin{array}{lll}
1 & \left(T_{i}-T_{i-1}\right)<0 & \frac{100}{n_{2}} \sum_{i=1}^{n} d_{i}, \\
0 & \text { Other } & \left(P_{i}-P_{i-1}\right)\left(T_{i}-T_{i-1}\right) \leq 0
\end{array}\right.
\end{gathered}
$$

where $T$ is the actual value, $P$ is the predicted value, $n$ is the total number of samples, $n_{1}$ is the number of the samples with upward trend; $n_{2}$ is the number of the samples with downward trend.

Fig. 3 show the training convergence curves for 2RS-WNN In Table 2 4, compared with 3 NNs including BP-NN, RBF-NN and ANFIS-NN, 3 WNNs including WNN, RS-WNN and 2RS-WNN have the superiorities in training time, prediction error and prediction accuracy as a whole, which is consistent with the conclusion of theoretical analysis and numerical calculation. In 3 WNN models, the overall performance of RS-WNN model is better than that of simple WNN model. DS value corresponding to SSE Composite Index, CSI 300 Index and All Ordinaries Index is $64.01 \%$, 66.24\% and $65.80 \%$ respectively, which has an improvement of 3.78\%, $5.73 \%$ and $3.45 \%$ in contrast with simple WNN model. It shows that, through RS attribute reduction, the redundant information in 15 index feature data of shock price trend is largely eliminated, reducing the input dimension of $\mathrm{WNN}$, which can effectively improve the prediction perfor- mance of the model. For 2RS-WNN model, Its DS value corresponding to SSE Composite Index, CSI 300 Index and All Ordinaries Index is $65.75 \%, 66.37 \%$ and $65.93 \%$ respectively, and only increases $0.74 \%, 0.13 \%$ and $0.17 \%$ comparing with RS-WNN model, however, the training time saves the $2.5 \mathrm{~s}, 2.3 \mathrm{~s}$ and $3.6 \mathrm{~s}$ respectively. This shows that, using RS to reduce the hidden layer nodes on the basis of RS-WNN, can essentially optimize the network structure of WNN. This can greatly shortens the training time with slight accuracy improvement, which is an important perfor- mance index for the incremental modeling and will greatly promote the actual application of proposed 
method. In summary, 2RS-WNN can greatly shorten the training time of prediction model and improve the prediction accuracy for stock price trend, and the obtained prediction results also can accurately reflect the overall trend of the stock market.

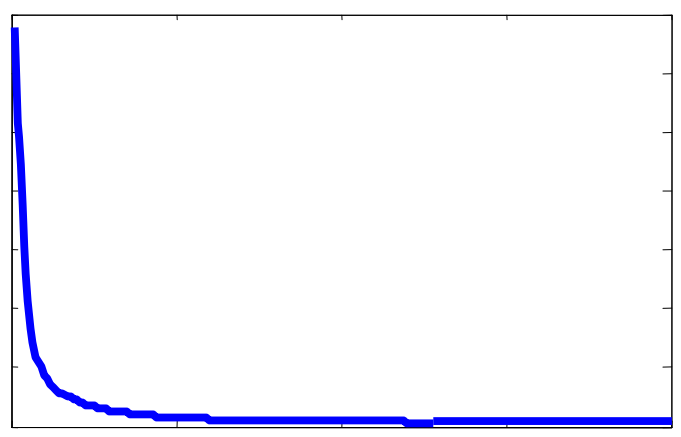

Fig. 3. Training convergence curves for $2 \mathrm{RS}-\mathrm{WNN}$

TABLE II. PERFORMANCE COMPARISON BETWEEN DIFFERENT MODELS FOR SSE COMPOSITE INDEX

\begin{tabular}{ccccccc}
\hline Index & BP-NN & RBF-NN & $\begin{array}{c}\text { ANFIS- } \\
\text { NN }\end{array}$ & WNN & RS-WNN & $\begin{array}{c}\text { 2RS- } \\
\text { WNN }\end{array}$ \\
\hline $\begin{array}{c}\text { Model } \\
\text { structure }\end{array}$ & $15-12-1$ & $15-7-1$ & $15-8-1$ & $15-8-1$ & $8-7-1$ & $8-5-1$ \\
$\begin{array}{c}\text { Training } \\
\text { time(s) }\end{array}$ & 14.3 & 9.7 & 12.4 & 5.5 & 4.2 & 1.7 \\
RMSE & 216.25 & 175.74 & 153.22 & 135.38 & 107.64 & 98.50 \\
MAD & 192.54 & 141.88 & 137.72 & 125.18 & 92.05 & 77.18 \\
MAPE & 4.41 & 3.26 & 3.54 & 3.15 & 1.97 & 1.75 \\
DS\% & 59.80 & 61.08 & 61.58 & 60.23 & 64.01 & 65.75 \\
CP\% & 61.55 & 59.17 & 62.11 & 62.24 & 63.48 & 66.73 \\
CD\% & 58.36 & 62.79 & 58.45 & 58.35 & 65.77 & 65.91 \\
\hline
\end{tabular}

TABLE III. PERFORMANCE COMPARISON BETWEEN DIFFERENT MODELS FOR CSI 300 INDEX

\begin{tabular}{ccccccc}
\hline Index & BP-NN & RBF-NN & $\begin{array}{c}\text { ANFIS- } \\
\text { NN }\end{array}$ & WNN & RS-WNN & $\begin{array}{c}\text { 2RS- } \\
\text { WNN }\end{array}$ \\
\hline $\begin{array}{c}\text { Model } \\
\text { structure } \\
\text { Training } \\
\text { time (s) }\end{array}$ & $15-10-1$ & $15-7-1$ & $15-6-1$ & $15-8-1$ & $7-9-1$ & $7-5-1$ \\
RMSE & 167.40 & 148.09 & 142.25 & 115.02 & 97.24 & 84.25 \\
MAD & 133.21 & 124.01 & 117.05 & 75.18 & 59.03 & 45.12 \\
MAPE & 5.94 & 5.26 & 3.54 & 2.87 & 2.66 & 1.84 \\
DS\% & 58.54 & 59.08 & 59.87 & 60.51 & 66.24 & 66.37 \\
CP\% & 57.14 & 60.22 & 57.14 & 62.70 & 65.48 & 67.25 \\
CD\% & 60.15 & 58.38 & 60.15 & 59.84 & 66.77 & 66.18 \\
\hline
\end{tabular}

TABLE IV. PERFORMANCE COMPARISON BETWEEN DIFFERENT MODELS FOR ALL ORDINARIES INDEX

\begin{tabular}{ccccccc}
\hline Index & BP-NN & RBF-NN & $\begin{array}{c}\text { ANFIS- } \\
\text { NN }\end{array}$ & WNN & RS-WNN & $\begin{array}{c}\text { 2RS- } \\
\text { WNN }\end{array}$ \\
\hline $\begin{array}{c}\text { Model } \\
\text { structure }\end{array}$ & $15-14-1$ & $15-9-1$ & $15-10-1$ & $15-6-1$ & $6-8-1$ & $6-3-1$ \\
$\begin{array}{c}\text { Training } \\
\text { time (s) }\end{array}$ & 16.2 & 9.5 & 12.0 & 6.4 & 5.7 & 2.1 \\
RMSE & 239.14 & 180.23 & 165.25 & 127.85 & 106.85 & 97.55 \\
MAD & 207.79 & 145.10 & 122.43 & 105.12 & 84.80 & 70.23 \\
MAPE & 3.95 & 3.34 & 1.32 & 1.17 & 4.08 & 1.99 \\
DS\% & 60.74 & 61.95 & 61.27 & 62.35 & 65.80 & 65.97 \\
CP\% & 63.78 & 60.90 & 60.90 & 61.54 & 64.27 & 64.32 \\
CD\% & 57.89 & 63.44 & 63.44 & 64.02 & 66.56 & 67.41 \\
\hline
\end{tabular}

\section{CONCLUSION}

Stock market is an important part of the economic system. The evolution process of stock price is decided by many economic individuals and economic factors working together, resulting in great uncertainty, which brings difficulties to the stock price prediction. For that reason, an integrated prediction method based on RS and WNN is proposed. It provides an effective solution for the prediction of stock price trend. It is believed that, with further research, through selecting more reasonable index, the prediction accuracy can further be improved for stock price trend. The inadequacy of this paper is: using 4 year data to establish the model may cause time delay, which will affect the prediction effect for stock price trend. To solve this issue, the introduction of a forward rolling EMD technology in 2RS-WNN modeling will be the next study focus.

\section{REFERENCES}

[1] M. T. Philp, K. Paul, S. O. Choy, "Design and implementation of NNS for Hong Kong stock price forecasting," Engineering Applications of Artificial Intelligence, vol. 20, No. 4, 2007, pp. 453-461.

[2] A. L. Leone, A. S. F. Ricardo, G. L. Guilherme , "Maximum and minimum stock price forecasting of Brazilian power distribution companies based on artificial neural networks," Applied Soft Computing, vol. 35, 2015, pp. 66-74.

[3] Q. H. Zhang, A. Benveniste, "Wavelet network," IEEE Trans- actions on Neural Networks, vol. 3, No. 6, 1992, pp. 889-898.

[4] Q. H. Zhang, "Using wavelet network in nonparametric estimation," IEEE Transactions on Neural Networks, vol. 8, No. 2, 1997, pp. 227-236.

[5] Z. Pawlak, "Rough Sets," International Journal of Computer and Information Sciences, vol. 1, No. 11, 1982, pp. 341-356.

[6] Z. Pawlak, "Rough sets-theoretical aspects of reasoning about data," Dordrecht: Kluwer Academic Publisher, 1990.

[7] B. B. Qu, Y. S. Lu, "Rough set-based algorithm for attribute reduction," Journal of Huazhong University of Science and Technology, vol. 33, No. 8, 2005, pp. 30-30.

[8] B. F. Wu, Q. Li, W. Z. Song, "Inductive learning approach to knowledge representation system based on rough set theory," Control and Decision, vol. 14, No. 3, 1999, pp. 206-211.

[9] L. Zhu, J. M. He, S. Chang. Rough set integrated neural network model for forecasting stock price," Chinese Journal of Management Science, vol. 10 , No. 4,2002 , pp. 7-12. 